\title{
INTERDISCIPLINARIEDAD: DESAFÍO PARA LA EDUCACIÓN SUPERIOR Y LA INVESTIGACIÓN
}

\author{
YESID CARVAJAL ESCOBAR ${ }^{1}$ \\ yecarvaj@univalle.edu.co
}

Manizales, 2010-04-21 (Rev. 2010-05-11)

\section{RESUMEN}

En los últimos años, los problemas globales han aumentado en complejidad y conectividad, especialmente los ambientales (crisis del agua, cambio climático, demanda de energía, crecimiento poblacional, etc.), lo que obliga a enfocarlos como complejos, inseparables y retroalimentados. La sectorización del pensamiento, trabajo e indicadores de rendimiento (enfocados generalmente a indicadores económicos), son obstáculos para alcanzar metas más integrales, intensificándose el trabajo fraccionado, la especialización, el enfoque sectorial y el individualismo sobre el enfoque sistémico.

A partir de lo anterior, surge la necesidad de implementar una visión holística, integral e interdisciplinaria para resolver los problemas actuales, que sólo se logra con el trabajo en equipo. En este artículo, se describen los diferentes conceptos de abordaje del conocimiento y se plantea la necesidad de hacer cambios en la educación superior y la investigación, para abordar estos problemas cada vez más complejos. Se concluye que el desarrollo humano sostenible puede abordarse desde diferentes disciplinas, pero ninguna por sí sola podrá responder a sus principales problemas, siendo muy poco lo que aportan los equipos multidisciplinarios si los expertos de cada disciplina ofrecen sólo una visión técnica de su especialidad, sin articular el conocimiento con las demás.

\section{PALABRAS CLAVE:}

Interdisciplinariedad, multidisciplinariedad, transdisciplinariedad, pensamiento complejo, enfoque sistémico.

\section{INTERDISCIPLINARITY: A CHALLENGE FOR HIGHER EDUCATION AND RESEARCH}

\begin{abstract}
In recent years, global problems have increased in complexity and connectivity, especially those environmental (water crisis, climate change, energy demand, population growth, etc.) which forces to focus them as complex, inseparable, and feed backed. Thought sectioning and work and performance indicators (generally focused on economical indicators) are obstacles for the achievement of more comprehensive goals, intensifying in divided work, specialization, sectional focus and individualism over the systemic approach. From this premise it appears the need to implement a holistic, comprehensive, and interdisciplinary vision in order to solve current problems which can only be achieved through team work. In this article the different concepts to deal with knowledge are described and the need to make some changes in higher education and research processes to face these everyday more complex problems is considered. It is concluded that sustainable
\end{abstract}


human development can be dealt with from different disciplines, but none by itself will answer its main problems being not much what interdisciplinary teams contribute if the experts in each discipline offer only a technical vision of their specialty without articulating knowledge with the others.

KEY WORDS: interdisciplinarity, multidisciplinarity, transdisciplinarity, complex thinking, systemic approach.

\section{INTRODUCCIÓN}

Urgentemente, la sociedad reclama de la Ciencia \& Tecnología \& Innovación, una investigación orientada a la solución de problemas y aportes verificables en respuesta a sus necesidades. Para Colombia, un país con grandes desafíos sociales, económicos y ambientales tales como: hambre, desnutrición, pobreza extrema, desempleo, violencia y deterioro ambiental, entre otros, esto se constituye en un reto para la comunidad científica y tecnológica (Sánchez, 2007).

La actual tendencia de desarrollo mundial no es sostenible, y las necesidades de una población en crecimiento en un mundo desigual e interconectado, están debilitando los sistemas naturales de apoyo vital del planeta. La globalización y los cambios económicos mundiales, entre otros, han dinamizado el desarrollo de la tecnología, la información, el intercambio comercial; a lo cual se adiciona el aumento de la población y el consumismo (Tiessen, 2008). Como resultado, ha aumentado la complejidad y conectividad de los problemas, en especial los ambientales (crisis del agua, cambio climático, demanda de energía, crecimiento poblacional, etc.) que hacen que estos sean menos disgregables y obliga a enfocarlos como complejos, inseparables y retroalimentados; requiriéndonos, e incluso exigiéndonos, reformas en la educación y la investigación con un enfoque interdisciplinario (Nunes, 2002; CEPAL-ONU, 2003; Restrepo, 2004; Carvajal, 2008a, 2008b; Tiessen, 2008).

La sectorización del pensamiento, trabajo e indicadores de rendimiento (enfocados generalmente a indicadores económicos), y la división arbitraria de territorios, son obstáculos para alcanzar metas integrales, que intensifican el trabajo fraccionado, sectorial e individualizado sobre el enfoque sistémico. Los errores que se cometen con los enfoques fraccionados en la gestión del talento humano, han ocasionado mayores demandas de coordinación en la toma de decisiones.

En la gestión de los recursos naturales, el enfoque eco-sistémico se viene consolidando como una estrategia que integra las diferentes dimensiones del desarrollo. Los ecosistemas son reguladores importantes de la cantidad y calidad de agua; y para realizar estas funciones, requieren protección y gestión, sin las cuales ocurrirían serias consecuencias ambientales, sociales y económicas. Por tanto, es evidente que para conseguir la gestión sostenible de los recursos hídricos y funciones eco-sistémicas, incluyendo las necesidades humanas, se necesita un enfoque integral. En ese sentido, la interdisciplinariedad puede ser un logro que trasciende a las profesiones, y un medio para construir una sociedad más justa y humana. El enfoque eco-sistémico es una estrategia para la gestión integral de suelo, agua y recursos vivos que promueve la conservación y el uso sostenible de una manera equitativa y complementa el pensamiento actual sobre la Gestión Integral del Recurso Hídrico; ambos conjuntos de principios son consistentes entre sí (Restrepo, 2004; Guerrero, De Keizer \& Córdoba, 2006; García, Carvajal \& Jiménez, 2007; Carvajal, 2008a, 2008b, 2009). 
La visión holística e integral permite resolver los problemas ambientales actuales, mediante el trabajo en equipo que implica un abordaje interdisciplinario y transdisciplinario. Según la CEPAL-ONU (2003), los problemas modernos deben estudiarse desde varias disciplinas y conformando equipos, para obtener soluciones integrales, dado que ninguna disciplina, por separado, puede dar resultados por sí mismas (García, s.f.). Los equipos interdisciplinarios pueden aportar muy poco, si contribuyen únicamente con una visión técnica, sin integrar su conocimiento con las demás disciplinas.

El paso de la multidisciplinariedad a la interdisciplinariedad y/o a la transdisciplinariedad (Posada, 2004; Max-Neef, 2005), requiere del desarrollo de metodologías de trabajo en equipo y de integración entre diferentes ciencias (incluso especialidades de una misma ciencia) que, específicamente aplicadas a las disciplinas, puedan contribuir al desarrollo sostenible. El entorno histórico actual es muy diferente del pasado relativamente reciente; el mundo está concurriendo hacia un contexto globalizado; la interconexión creciente de los sistemas naturales y sociales, y la progresiva complejidad de las sociedades y de sus impactos sobre el planeta, generan altos niveles de incertidumbre (Gallopín, Funtowicz, O'Connor \& Ravetz, 2001). Un alto porcentaje de la población colombiana se ha empobrecido en los últimos años y vive en ambientes deteriorados; en este contexto, la complejidad de los problemas y situaciones seguirá aumentando rápidamente en las décadas venideras.

\section{DEFINICIONES DE DISCIPLINA, MULTIDISCIPLINA, INTERDISCIPLINA, TRANSDISCIPLINA}

Las múltiples problemáticas del siglo XXI, tienen en común la complejidad y que ninguna de ellas puede abordarse desde el ámbito individual de las disciplinas, si no que se son desafíos transdisciplinarios (Max-Neef, 2005). Esto no generaría preocupaciones si la formación de capacidades en las universidades fuese acorde con tal reto, pero infortunadamente sigue dominando la enseñanza sectorizada, con pocas excepciones. Ciertos autores sitúan la interdisciplinariedad dentro de un conjunto de términos: multidisciplinariedad, pluridisciplinariedad, y transdisciplinariedad (Zárate, 2007), que invitan necesariamente a una aclaración de conceptos y de términos:

\subsection{Disciplinariedad}

La disciplinariedad es mono-disciplina, que representa especialización en aislamiento (Max-Neef, 2005); concierne más o menos a un sólo y mismo nivel de realidad, contraria a la transdisciplinariedad, que se interesa en la dinámica que se genera de la acción simultánea de varios niveles de realidad (Valle, 2005).

\subsection{Multidisciplinariedad}

Es una mezcla no-integradora de varias disciplinas, en la que cada una conserva sus métodos y suposiciones sin cambio o desarrollo de otras disciplinas (Rodríguez, s.f.). Se entiende como el trabajo indagatorio concurrente de varias disciplinas diferentes, hacia el encuentro de un mismo problema (métodos, desarrollos conceptuales) con otras disciplinas (Sotolongo \& Delgado, 2006). Los investigadores acogen las relaciones de colaboración con objetivos comunes; hacen sus análisis independientes, y en el resultado final no presentan un 
resumen integrador, ni establecen vínculos entre disciplinas, ni una perspectiva articulada. Posada (2004), destaca que es el nivel inferior de integración, que ocurre cuando alrededor de un interrogante se busca información y ayuda en varias disciplinas, sin que dicha interacción contribuya a modificarlas o enriquecerlas. Esta puede ser la primera fase de la constitución de equipos de trabajo interdisciplinario. Se diferencia de la interdisciplinariedad, en que en una relación multidisciplinar esta cooperación puede ser mutua y acumulativa pero no interactiva (Augsburg, 2005); por su lado, la interdisciplinariedad mezcla las prácticas y suposiciones de las disciplinas implicadas, con una mayor integración entre ellas (Sánchez, 2002).

\subsection{Pluridisciplinariedad}

Implica cooperación entre disciplinas, sin coordinación; normalmente se da entre áreas del conocimiento compatibles entre sí, y de un mismo nivel jerárquico (Max-Neef, 2005). Rodríguez (s.f.) destaca que la pluridisciplinariedad establece determinada relación entre los saberes participantes, pero una de ellas lidera estas relaciones, plantea los términos de tal relación y el método de los procesos se rige por el rigor de dicha disciplina. Torres (1994), por su parte, la define como la unión no-integrativa de dos o más disciplinas, más o menos cercanas y por lo general dentro de un campo de conocimientos, que conservan sus métodos y modelos propios, como ocurre en la multidisciplinariedad, pero en la que se busca mejorar la relación entre ellas. Esto implica la existencia de relaciones de colaboración entre las diferentes disciplinas, evidencia objetivos comunes; y presupone una perspectiva de complementariedad entre las disciplinas, sin la existencia de sistematización o integración (Jauregui, s.f.).

\subsection{Interdisciplinariedad}

La literatura está llena de diferentes definiciones de interdisciplinariedad y cada una de ellas asume las especificidades del contexto en que son usadas (Lattuca, 2001). La interdisciplinariedad puede verse como una estrategia pedagógica que implica la interacción de varias disciplinas, entendida como el diálogo y la colaboración de éstas para lograr la meta de un nuevo conocimiento (Van del Linde, 2007). De otro lado, Sotolongo y Delgado (2006) la definen como el esfuerzo indagatorio y convergente entre varias disciplinas (en ese sentido, presupone la multidisciplinariedad) pero que persigue el objetivo de obtener "cuotas de saber" acerca de un objeto de estudio nuevo, diferente a los que pudieran estar previamente delimitados disciplinaria o multidisciplinariamente. Posada (2004), la define como el segundo nivel de integración disciplinar, en el cual la cooperación entre disciplinas conlleva interacciones reales; es decir, reciprocidad en los intercambios y, por consiguiente, un enriquecimiento mutuo. En consecuencia, se logra una transformación de conceptos, metodologías de investigación y de enseñanza. Implica también, a juicio de Torres (1996), la elaboración de marcos conceptuales más generales, en los cuales las diferentes disciplinas en contacto son a la vez modificadas y pasan a depender unas de otras. La interdisciplinariedad cobra sentido en la medida en que flexibiliza y amplía los marcos de referencia de la realidad, a partir de la permeabilidad entre las verdades de cada uno de los saberes (Follari, 2007; Rodríguez, s.f.).

\subsection{Transdisciplinariedad}

Es la etapa superior de integración disciplinar, donde se llega a la construcción de sistemas teóricos totales (macro-disciplinas o trans-disciplinas), sin fronteras sólidas entre las disciplinas, fundamentadas en objetivos comunes y en la unificación epistemológica y cultural (Posada, 2004; Stokols, 2006). La 
transdisciplinariedad posibilita la articulación de otros marcos, al proceso de conocimiento específico de una disciplina, de tal forma que podría decirse que en la actualidad los paradigmas de una ciencia o saber no le pertenecen exclusivamente, y es necesario extrapolarlos a diferentes contextos teóricos y metodológicos (Nicolescu, 1998, 2002; Rodríguez, s.f.). En cuanto a principios de formas integradoras de investigación, la transdisciplinariedad comprende una familia de métodos para relacionar el conocimiento científico, la experiencia extra-científica y la práctica de la resolución de problemas. En esta comprensión, la investigación transdisciplinar se orienta hacia los aspectos del mundo real, más que a aquellos que tienen origen y relevancia sólo en el debate científico. Una cuestión de mayor importancia en este tipo de investigación es: hasta qué punto se consigue la integración de las distintas perspectivas científicas. Este aspecto es a menudo usado para distinguir entre trans, ínter y multidisciplinariedad.

\section{LOS NUEVOS ENFOQUES EN LA EDUCACIÓN SUPERIOR (ES)}

\subsection{Enfoque tradicional}

En el siglo $X X I$, los desafíos de la ciencia son de tal magnitud que, de no ser atendidos con oportunidad y eficacia, ahondarán las diferencias, desigualdades y contradicciones que hoy condicionan el crecimiento con equidad, justicia, sustentabilidad y democracia para América Latina y el Caribe (CRES, 2008). La Educación Superior (ES) es la base para el papel estratégico que deben jugar los procesos de desarrollo sustentable de los países de la región. Como parte de su responsabilidad social, la ES debe poner en su agenda temas urgentes que enfrenta la humanidad, como son los problemas ambientales, interculturales, etc. Esto impone la necesidad de orientar los conocimientos técnico-científicos, y toda la capacidad crítica en la perspectiva de los ideales humanitarios de libertad, justicia social, paz y desarrollo humano (Vessuri, 2008). En este sentido, la interdisciplinariedad, más que un término, debe ser una estrategia pedagógica, que implica conceptualizar los propósitos y la planificación del proceso, con una previa evaluación del sistema educativo como punto de partida (Zárate, 2007).

La adquisición de habilidades y competencias en el modelo tradicional de ES es muy conservadora y contribuye poco al logro de altos niveles de desempeño e innovación (Capper, 2001). Actualmente los problemas desafían los modelos individualistas y tradicionales relacionados con las habilidades. El manejo y análisis en equipo de la información es más importante que llevarlos a cabo individualmente. Por tal razón, el trabajo colectivo es más trascendental que la contribución individual (Capper, 2001).

Los conceptos influyentes en competencias laborales son caducos, porque se fundamentan en: a) la adquisición de habilidades individuales, sin considerar las organizacionales y el trabajo en equipo; b) en la educación y capacitación formales, sin tener en cuenta el aprendizaje cotidiano producto del trabajo, clave para la optimización del aprendizaje y el perfeccionamiento continuo; c) en los niveles de habilidad de los empleados nuevos o sin trabajo, sin considerar los conocimientos, valores y habilidades que requieren los gerentes, los supervisores y demás personal responsable de facilitar el aprendizaje continuo. Autores como Morin (1999) resaltan que “... existe una inadecuación cada vez más amplia, profunda y grave de un lado, nuestros saberes desarticulados, parcelados y compartimentados $\mathrm{y}$, por el otro, las realidades o problemas cada vez más 
complejos, multidimensionales, polidisciplinarios, transversales, transnacionales, globales, planetarios..." (pág 14).

\subsection{Enfoque moderno}

Desde esta perspectiva integral, se requiere redefinir las habilidades y competencias, considerando aspectos tales como:

La formación con base en competencias, que conlleva integrar disciplinas, conocimientos, habilidades, prácticas y valores. La integración disciplinar es parte fundamental de la flexibilización curricular, particularmente de los planes de estudio, con el fin de formar profesionales más universales, aptos para afrontar los rápidos cambios de las competencias y los conocimientos; con una formación más humanística y ambiental, con ética, conciencia de equidad social y juicio crítico, que actúen como agentes de cambio social, dada la importancia de su trabajo para mejorar la calidad de vida de los más pobres, bajo los lineamientos del desarrollo sostenible (Restrepo, 2004). Lo que se pretende, más que generalizar como dicen los críticos del enfoque interdisciplinario, es simplificar el lenguaje de interdicción entre disciplinas.

Para que la investigación se mantenga, es sustancial mantenerla unida a la docencia; ambas se alimentan recíprocamente, por lo que es improductivo separarlas o privilegiar una sobre la otra. La investigación abre nuevos horizontes al alumno, completa la información ofrecida por los docentes y permite una especialización personal enriquecedora. Agazzi (2001) propone las siguientes orientaciones metodológicas para la investigación interdisciplinar:

- La investigación interdisciplinar debe partir de la individualización del problema y de los aspectos del mismo que requieren del enfoque de disciplinas bien individualizadas.

- Establecer claramente los distintos criterios disciplinares para abordar los datos.

- Explicitar el marco teórico de las disciplinas, sin cuestionar sus supuestos.

- Definir los conceptos empleados por las distintas disciplinas involucradas, relacionándolos con su marco conceptual y su metodología de acceder a los datos.

- Aceptar la autonomía de los procedimientos lógicos de las disciplinas.

Ackerman (1988) por su parte, plantea que la interdisciplina ofrece a los estudiantes los siguientes beneficios: Contribuye a generar pensamiento flexible, desarrolla habilidad cognitiva de alto orden, mejora habilidades de aprendizaje, facilita mejor entendimiento de las fortalezas y limitaciones de las disciplinas, incrementa la habilidad de acceder al conocimiento adquirido y mejora habilidades para integrar contextos disímiles. Adicionalmente, permite sintetizar e integrar para producir originalidad; también aumenta el pensamiento crítico y desarrolla la humildad, al tiempo que empodera y desmitifica expertos (Field, 1994).

Las actividades académicas de integración disciplinar contribuyen a afianzar valores en profesores y estudiantes: flexibilidad, confianza, paciencia, intuición, pensamiento divergente, sensibilidad hacia las demás personas, aceptación de riesgos, movilidad en la diversidad, y aceptación de nuevos roles, entre otros (Torres, 1996). 


\section{DESAFÍOS EN EL ABORDAJE DEL TRABAJO INTERDISCIPLINARIO}

El trabajo interdisciplinario presenta múltiples desafíos para abordar; entre ellos se tienen:

\subsection{El lenguaje científico}

En cada disciplina éste es diferente (términos, ecuaciones, conceptos, etc.); frecuentemente confunde a los científicos de otras disciplinas; y en ocasiones, las palabras clave y los conceptos tienen significados disímiles según la disciplina. Así mismo, en otros idiomas, las palabras normales tienen significados disciplinarios que no son muy comunes. La investigación interdisciplinaria debe salvar estas barreras, buscar un lenguaje común y simplificarlo, para trabajar con él, o al menos aceptarlo (Juarroz, 1996; Luckman, 2008; Rodríguez, s.f.).

\subsection{Egocentrismo intelectual}

Una de las principales barreras en el trabajo interdisciplinario está relacionada con el egocentrismo intelectual y el hermetismo del pensamiento, que se protege y confina para evitar ser cuestionado o enriquecido desde otra perspectiva (Rodríguez, s.f.). Una clave para el diálogo interdisciplinario productivo, consiste en la definición de los problemas tal como se ven por parte de los especialistas, reconociendo los límites de las soluciones y conocimientos que pueden ofrecer. Varias disciplinas tienen su propio conjunto de normas para abordar o formular problemas y no reconocen otras. Por tanto, se requiere cooperación, respeto mutuo, confianza y apertura mental para manifestar las complementariedades y lo que cada uno puede aportar a un proyecto interdisciplinario. La crítica destructiva desde una disciplina en particular no contribuye a la construcción de puentes entre ellas. Aquí se requiere trabajar conjuntamente, reconocer limitaciones, estar abierto a críticas y tener voluntad de aprender de otros, resultando de esto la necesidad de establecer equipos de trabajo, con el fin de cooperar; porque la misma complejidad de los problemas lo exige. Para ello se requieren personas con capacidad de flexibilidad, confianza, paciencia, intuición, pensamiento divergente, sensibilidad hacia los demás, moderación, mediación, asociación y transferencia, entre otros; con el fin de iniciar y promover un diálogo constructivo, crítico y permanente (Zárate, 2007). La investigación interdisciplinaria frecuentemente requiere más tiempo, esfuerzo, imaginación y tiene más riesgo de fracaso que la unidisciplina, no obstante la retribución es importante en el avance de la base de conocimientos y la resolución de problemas sociales complejos (Wallerstein, 1996).

Expandirse al trabajo interdisciplinario parece ser sencillo, pero el interés de los investigadores por llevar a cabo el proyecto puede limitarse por la tendencia a fortalecer el área específica de su formación. Así, muchos trabajos interdisciplinarios se hacen entre disciplinas tan afines, que generan la duda de si realmente se trata de otras disciplinas (Cabrera, 2006).

\subsection{Estructuras institucionales y procedimientos}

La interdisciplinariedad debe ser un objetivo tanto para los nuevos científicos, como para los educadores; dado que está relacionada con la percepción de la ciencia por parte del público, y especialmente con el modo en que la integración de las distintas ciencias puede mejorar la comprensión y la búsqueda de soluciones a problemas ambientales. Esto requiere, desde la educación e investigación, incentivar el trabajo en equipos interdisciplinarios 
A nivel de educación existen barreras a la interdisciplinariedad, entre las cuales se cuentan: sistemas académicos rígidos, asimetría entre los campos de conocimiento dentro de las instituciones, y largos procesos de aprobación de nuevos planes de estudio que se construyen sobre conceptos novedosos de integración entre distintas ciencias. La interdisciplinariedad puede introducirse en los programas disciplinarios tradicionales, pero esto ocurre más por interacciones directas entre profesores de diferentes disciplinas, que a través de una iniciativa estructurada de las autoridades universitarias (Bustamante, 2008).

Concepción sistémica. Introducir la interdisciplinariedad en la universidad y en la investigación requiere un ejercicio analítico de su verdadero significado; y conlleva un acuerdo previo de concebir la realidad en su diversidad y variabilidad, en contraste a una cosmovisión estricta y sencilla; por esto, se plantean dos requerimientos básicos: lograr una "cosmovisión", una mentalidad sistémica, de globalidad y complejidad acerca de los distintos saberes, pero que integre sus especializaciones y especificidad en cuanto a la formación; y en segundo lugar, una interdisciplinariedad para nuevas creaciones científicas y teóricas que logren plantear alternativas de solución a los problemas reales de la sociedad (Pedroza \& Argüello, 2002; Follari, 2005).

\subsection{Publicaciones especializadas}

Otro desafío básico se relaciona con la sociología de la ciencia. Frecuentemente, para avanzar en la disciplina elegida, los científicos principiantes deben seguir la regla no escrita de publicar en revistas que son de su disciplina, o caso contrario, se considera publicación en revistas de menor importancia. La idea de que la interdisciplina es una ciencia blanda y que no cuenta, porque no se ajusta a las convenciones de las disciplinas o se publica en revistas de menor importancia, es una idea aún por revaluar, hasta tanto se comprenda verdaderamente el trabajo interdisciplinario y los diferentes enfoques y puntos de vista (Luckman, 2008). Muchas veces se confunde que la interdisciplina es una ciencia blanda y no tiene validez, por no ajustarse a los estándares disciplinarios, pero este imaginario de creer que está en peligro la profundidad del conocimiento, no tiene validez porque por el contrario, permite fortalecerlas por el abordaje de los problemas desde perspectivas diferentes, lo cual ayuda a entender temas que no son claramente visualizados desde una sola perspectiva (Van del Linde, 2007).

\subsection{Tiempos diferentes}

El trabajo interdisciplinario ofrece un aporte significativo a la academia; si bien los horizontes temporales de los estudios científicos, políticos y sociales, generalmente son diferentes y complicados de ajustar, es posible la cooperación, con voluntad, paciencia y comprensión. Mucho se ha escrito sobre la necesidad de una investigación interdisciplinaria, y muchos científicos en el mundo se esfuerzan por aplicarla con mayor o menor éxito. Un investigador podría cuestionarse por qué, después de formarse en unidisciplina, se espera que aborde un enfoque integral; una de las razones es la globalización, que impone nuevas demandas y normas a la ciencia y su interrelación con la sociedad (Tiessen, 2008). Esto hace que el conocimiento y la comprensión para la toma de decisiones, sean complejos y vayan más allá de las capacidades de las disciplinas individuales; significa comprometer a científicos de diferentes disciplinas (ciencias naturales y sociales), para que brinden análisis, síntesis y pronósticos a la sociedad, a los responsables de formulación de políticas y a los tomadores de decisión. 


\section{LA INGENIERÍA Y LA INTERDISCIPLINARIEDAD}

La formación en ingeniería todavía está lejos de ser interdisciplinaria y mucho más de ser transdisciplinaria; se espera que la ingeniería sea un instrumento para el mejoramiento de la calidad de vida del ser humano sin deteriorar la calidad ambiental, siguiendo los lineamientos del Desarrollo Sostenible. Por esto, las concepciones sectoriales en la ingeniería deberán cambiar para acoger todos estos conceptos que, se espera, contribuyan a la equidad y la protección de los recursos ambientales (Restrepo, 2004). En el caso del agua, la escasa visión integral ofrecida por las escuelas de ingeniería tradicionales, se reconoce como uno de los fracasos en la educación, orientada sólo a aspectos técnicos (Cairncross, 1992; Kolsky \& Cotton, 1996; Carvajal, 2008a). Mejía (2004) en consulta realizada a 40 expertos locales en 5 países de América Latina y el Caribe, destaca que hay consenso en que los programas de estudio están obsoletos y fuera de contexto de los desarrollos y situaciones actuales. Se requiere un acercamiento a los aspectos sociales, económicos y culturales, que no se facilita durante los cursos de formación profesional. En consecuencia, la educación ofrecida no corresponde a las realidades observadas; resultados similares son reportados por Rodic y Sah (2005) y Mejía y Rodic-Wiesrma (2005).

Implementar la interdisciplinariedad en las instituciones de ES, implica tener en cuenta algunos aspectos como: eliminar fronteras para permitir el trabajo entre disciplinas; las estructuras de las instituciones (planes de estudio, instalaciones) frecuentemente representan obstáculos, al igual que las formas de financiamiento; cuando hay escasos recursos humanos y económicos para la educación disciplinaria e interdisciplinaria, tiende a predominar la primera sobre la segunda. Muchos cursos de ciencias ambientales más que de un enfoque interdisciplinario, se componen de una colección de disciplinas. El trabajo académico integrado, también requiere formas de encuentro en equipo, el establecimiento de criterios para la integración y desarrollo de ideas para precisar conceptos, temas, disciplinas, prácticas y competencias a integrar; de igual forma, es necesario definir los tipos de relaciones entre las disciplinas; determinar los tiempos para desarrollar los temas, problemas, etc.; evaluar continua y formativamente el proceso y reunir toda la información posible sobre experiencias en este campo.

Un aspecto fundamental de la ES, es la docencia o transmisión de conocimientos, la cual se logra activamente y con aprendizajes más significativos, cuando se refiere al estudiante a una educación interdisciplinaria (Van del Linde, 2007). Para lograr estos propósitos, Posada (2004) recomienda aplicar y fomentar pedagogías que estimulen y favorezcan procesos y actividades que desarrollen la capacidad y actitud de aprender, investigar, construir e innovar; en consonancia con la dinámica de los cambios: aprender a trabajar en equipo, desarrollando autonomía intelectual y responsabilidad individual y colectiva; adquirir mejores niveles de autoestima para la libre discusión, la argumentación racional, las competencias comunicativas, socioafectivas y profesionales, entre otros; y desmitificar la ciencia, el texto y el profesor como fuentes únicas de conocimiento. En consonancia, Posada (2004) señala que la docencia en la universidad debe ser ejercida por docentes estudiosos, competentes, comprometidos, conocedores de sus saberes y disciplinas, como también de su desempeño; sensibles a los cambios sociales e institucionales; sobre todo a las innovaciones pedagógicas y curriculares, de tal forma que logren las expectativas de sus estudiantes. 
La interdisciplinariedad y los ingenieros deben tener la capacidad de afrontar estos retos (CEPAL-ONU, 2003), porque se espera que las instituciones de ES respondan modernizando el contenido de sus programas académicos a los enfoques actuales (Carvajal, 2008a). Otro aspecto importante es la necesidad de un nuevo acercamiento a la evaluación de los estudiantes y a iniciativas tales como el trabajo en equipo, lo cual obliga a organizarse operacional e institucionalmente, para lograr la integración práctica. A pesar de las dificultades, hay iniciativas interesantes, específicamente en los programas de postgrado. Las instituciones de ES generalmente son más rígidas y conservadores con los programas de pregrado, presentando currículos ajustados; sin embargo, hay aspectos del aprendizaje y la enseñanza que impulsan la interdisciplinariedad (la enseñanza basada en problemas, el trabajo de campo y la cooperación) (Bustamante, 2008).

Una opción que favorece el proceso, es la movilidad estudiantil entre distintos programas universitarios, mediante la flexibilización de los programas educativos. El mundo exige nuevas demandas al modelo disciplinario, y no existe una solución simple y única; lo cual se convierte en una oportunidad para redefinir las universidades. La ES se mueve en nuevos escenarios, que demandan formas modernas de liderazgos gerenciales, para empresas y organizaciones de base científica y tecnológica, sustentadas fundamentalmente en la conformación de redes y alianzas, que permitan romper el aislamiento, socializar la información, acelerar la producción del conocimiento y equilibrar la transferencia de los mismos. El trabajo en redes es cada vez más importante, dada la casi imposibilidad de trabajar en forma aislada; adicionalmente, las redes permiten acelerar la transferencia de tecnología y la optimización del uso de recursos.

Los nuevos paradigmas de desarrollo que involucran la creciente demanda de una sostenibilidad ambiental, están fuertemente relacionados con cambios en la educación y la creación de nuevos perfiles profesionales que puedan integrar y elaborar el conocimiento de diversos campos, comunicarse, cooperar con diferentes actores, y actuar y moverse en equipos transfuncionales e intersectoriales; de hecho la ciencia ambiental es considerada una ciencia interdisciplinaria (Bustamante, 2008). Las universidades deben autotransformarse, para responder a las nuevas estructuras en red y generar bases de aprendizaje de alto valor social en los conocimientos desde una óptica interdisciplinaria y de investigación basada en el contexto de su aplicación, sin dejar de sustentar su visión crítica hacia la sociedad y su responsabilidad con el desarrollo humano y la sostenibilidad (Didriksson, 2008). Igual de importante, es la participación de la ES en la búsqueda de soluciones a los problemas humanos urgentes (Vessuri, 2008). A nivel universitario es necesario crear asignaturas y desarrollar programas interdisciplinarios, más flexibles con las expectativas del mundo actual; y permitir que la dinamización de la interdisciplinariedad progrese para beneficio de toda la humanidad (Van del Linde, 2007).

\section{CONCLUSIONES}

- Los problemas fundamentales que enfrenta la humanidad, obligan a estudiarlos como un todo, demandando el concurso de todas las potencialidades del conocimiento humano, y exigiendo enfocarlos como complejos, inseparables y retroalimentados; de tal forma que surge la necesidad de abordar una visión integral e interdisciplinaria para resolverlos, que plantea cambios en la educación y la investigación con nuevos enfoques ínter y transdisciplinarios. 
- Algunas de las principales barreras en el trabajo interdisciplinario se relacionan con la falta de un lenguaje común, el egocentrismo intelectual que impone cada disciplina como algo prioritario, las estructuras institucionales y procedimientos existentes; la falta de una concepción sistémica y sistemática de la complejidad de los distintos saberes y problemas, y la reticencia a reconocer que más que una ciencia blanda y superficial, la interdisciplina permite fortalecer las disciplinas por el abordaje de los problemas desde perspectivas diferentes, y es un medio para construir una sociedad más justa y humana.

- Lo transdisciplinario traspasa los límites de lo interdisciplinario y tiene como objetivo superar la sectorización del conocimiento, más allá del enriquecimiento de las disciplinas con diferentes saberes (multidisciplina) y del intercambio de métodos científicos de los saberes (interdisciplina). Refleja, especialmente, en el campo de la ES, la necesidad de permeabilización de las diferentes disciplinas, siendo un proceso en el cual los límites de las disciplinas individuales trascienden para estudiar problemas desde perspectivas múltiples para generar conocimiento.

- Existe incongruencia cada vez mayor entre los nuestros saberes desarticulados, y compartimentados y, por otro lado, los problemas cada vez más complejos y multidimensionales de la sociedad, que implican redefinir las habilidades y competencias en la ES y la investigación, con base en competencias, que integren disciplinas, conocimientos y habilidades. Al asumir la interdisciplinariedad en la ES, se requiere promover un enfoque de desarrollo, que además del crecimiento busque el desarrollo humano integral, formando continuamente estudiantes no sólo en sus habilidades o destrezas manuales, sino en valores solidarios y cooperativos.

- La interdisciplinariedad contribuye a generar pensamiento flexible, desarrolla y mejora habilidades de aprendizaje, facilita el entendimiento, incrementa la habilidad de acceder al conocimiento adquirido y mejora habilidades para integrar contextos disímiles. Así mismo, contribuye a afianzar valores en profesores y estudiantes, tales como: flexibilidad, confianza, paciencia, intuición, pensamiento divergente, sensibilidad hacia los demás y a aprender a moverse en la diversidad, entre otros.

- La ES es base para el papel estratégico que deben jugar los procesos de desarrollo sostenible, en los desafíos de la ciencia y los problemas ambientales, pero para ello es necesario establecer cambios en la misma y crear nuevos perfiles profesionales que puedan integrar y elaborar el conocimiento de diversos campos. En ese sentido, la interdisciplina, más que un término, debe ser una estrategia pedagógica, que conceptualice los propósitos y la planificación del proceso, con una previa evaluación del sistema educativo.

\section{RECONOCIMIENTOS}

Al Grupo de Investigación en Ingeniería de Recursos Hídricos y Desarrollo de Suelos -IREHISA- de la Escuela de Ingeniería de los Recursos Naturales y del Ambiente -EIDENAR- de la Universidad del Valle, por el apoyo con los recursos necesarios para la realización de este trabajo y por la experiencia adquirida en los proyectos interdisciplinarios. 


\section{REFERENCIAS}

- Ackerman, P. (1988). Determinants of individual differences during skill acquisition: Cognitive abilities and information processing. Journal of Experimental Psycology, 117(3), pp. 288-318. - Agazzi, E. (2001). El desafío de la interdisciplinaridad: dificultades y logros. Texto resumido del seminario para profesores impartido en el Departamento de Filosofía de la Universidad de Navarra. Obtenido el 28 de octubre de 2007, desde http://www.arvo.

net/pdf/El\%20desafio\%20de\%20la\%20interdisciplinaridad\%20dificultades\%20y\% 20logros.htm

- Andrade, A.; Navarrete, F. (2004). Lineamientos para la aplicación del enfoque ecosistémico en la Gestión integral del recurso hídrico. Ciudad de México: PNUMA. $110 \mathrm{p}$.

Augsburg, Tanya. (2005). Becoming Interdisciplinary: An Introduction to Interdisciplinary Studies. NY. Kendall/Hunt Pub. Co. p. 56. - Bustamante, Mercedes. (2008). Challenges of interdisciplinarity in universities. In $\quad I A I \quad$ Newsletterlssue $2 . \quad$ Disponible en http://www.iai.int/files/communications/newsletter/2008/issue_2_2008.pdf

- Cabrera, M. (2006). Trabajo Interdisciplinario. Ingenierías, abril-Junio, Vol. 9. No.

- Cairncross, S. (1992). Sanitation and Water Supply: Practical Lessons from The Decade. Washington: The World Bank. - Capper Phillip. (2001). La competencia en contextos laborales complejos. En Argüelles, Antonio y Gonczi, Andrew (eds), Educación y capacitación basada en normas de competencias: una perspectiva internacional. (pp 315). México: Limusa.

- Carvajal Escobar, Y. (2008a). Tendencias en la formación en ingeniería del agua en América Latina. Revista de la Escuela de Ingeniería de Recursos Naturales

$$
\text { y del }
$$

Ambiente

EIDENAR, (2008b). Environmental flow regime in the framework of integrated water resources management strategy. International Journal of Ecohydrology \& Hydrobiology, $\quad$ Vol. $\quad 8, \quad$ No. 2-4, 307-315. - (2009). Caudal ambiental: conceptos, experiencias y desafíos. Capítulo 1 del libro: Régimen de caudal ambiental en el marco de la gestión integrada del recurso hídrico. Cali: Programa Editorial Universidad del Valle. - CEPAL-ONU. (2003). Ciencia y tecnología para el desarrollo sostenible. Una perspectiva latinoamericana y caribeña. Taller Regional Latinoamericano y Caribeño sobre Ciencia y Tecnología para el Desarrollo Sostenible. Santiago de Chile, 5 al 8 de marzo de 2002. División de Desarrollo Sostenible y Asentamientos Humanos. Serie seminarios y conferencias No. 25. Santiago de Chile, enero de 2003. - CRES. (2008). Conferencia Regional de Educación Superior. Declaración final. Disponible http://www.iesalc.unesco.org.ve/index.php?option=com_content\&view=arti cle\&id=365\&ltemid $=423 \&$ lang $=e s$

- Didriksson, A. (2008). Contexto global y regional de la educación superior en América Latina y el Caribe. En Ana Lucía Gazzola y Axel Didriksson (Eds.), Tendencias de la Educación Superior en América Latina y el Caribe.,410pp. Caracas: IESALC-UNESCO.

- Field, M. (1994). Assessing Interdisciplinary Learning. New Directions for Teaching and Learning, 69-84. - Follari, R. (2005, Jun). La interdisciplina revisitada. Andamios, México, 1(2), 717.

- (2007). La interdisciplina en la Docencia. Polis, Revista académica http://www.revistapolis.cl/polis\%20final/16/follari.htm. 
- Gallopín, G.C., S. Funtowicz, M. O'Connor and J. Ravetz. (2001). Science for the 21st Century: from Social Contract to the Scientific Core. Int. Journal Social Science, 168 ,

219-229. - García, S. (s.f.). Hacia el sueño de la transdisciplinariedad. Obtenido en febrero de 2010, desde http://www2.minedu.gob.pe/digesutp/formacioninicial/?dl_id=32

- García, M., Carvajal, Y., Jiménez, H. (2007). La gestión integrada de los recursos hídricos como estrategia de adaptación al Cambio Climático. Ingeniería y competitividad, 9(1), 19-29. - Guerrero, E., De Keizer, O., Córdoba, R. (2006). La aplicación del enfoque ecosistémico en la gestión de los recursos hídricos. Quito, Ecuador: UICN. p. 78. - Jáuregui, Jorge Mario. (s.f.). Urbanismo y Transdisciplinariedad. Intersecciones (Puntuaciones en relación con el abordaje de la articulación de lo formal y lo informal en América Latina). Obtenido en marzo de 2009, desde http://www.jauregui.arq.br/transdisciplinariedad.html

- Juarroz, Roberto. (1996). Algunas ideas sobre el lenguaje de la transdisciplinariedad. Revista Complejidad, año 1, No. 2, junio-agosto. 19-21 pp. - Kolsky, P. \& Cotton A.P. (1996). Educating Engineers in Water and Sanitation. In Nabeel Hamdi, (Ed.), Educating for Real: the training of professionals for development practice. (Chapter 12: pp. 136-148). London: Intermediate Technology

Press.

- Lattuca, L. R. (2001). Creating Interdisciplinarity: Interdisciplinary Research and Teaching among College and University Faculty. Nashville, TN: Vanderbilt University

Press.

- Luckman, Brian. (2008). Some thoughts on interdisciplinarity from a CRN PIs perspective. In IAI Newsletterlssue 2. Disponible en http://www.iai.int/files/communications/newsletter/2008/issue_2_2008.pdf - Max-Neef, Manfred. (2005). Foundations of transdisciplinarity. Ecological Economics, $\quad 53, \quad$ 5-16. - Mejía Vélez, Diego. (2004). Postgraduate Capacities in the Water and Sanitation Sector in Latin America and the Caribbean: What do We Have? What do We Need to Achieve the MDGs? UNESCO-IHE, Delft. - Mejía Vélez, D.F. and Rodic-Wiesrma, L. (2005). Higher education in water and sanitation: An assessment in selected African countries. (draft paper). - Morin, Edgar. (1999). Los siete saberes necesarios para la educación del futuro. París: Organización de las Naciones Unidas para la Educación, la Ciencia y la Cultura. - Nicolescu, B. (1998). La transdisciplinariedad, una nueva visión del mundo. Manifiesto. París: Ediciones Du Rocher. - . (2002). Manifesto of Transdisciplinarity. Translation from the French by Karen-Claire Voss. New York, USA: State University of New York Press. - Nunes, E.D. (2002, Set-Dic). Interdisciplinariedad: conjugar saberes. Saúde em Debate, Río de Janeiro, Brasil, 26(62), 249-258. - Pedroza, R. y Argüello, F. (2002, Dic). Interdisciplinariedad y transdisciplinariedad en los modelos de enseñanza de la cuestión ambiental. Rev. Cinta de Moebio, 15. 3-16 pp. Facultad de Ciencias Sociales, Universidad de

Chile.

- Posada Álvarez, Rodolfo. (2004). Formación Superior basada en competencias, interdisciplinariedad y trabajo autónomo del estudiante, Revista Iberoamericana de Educación. Obtenido el 4 de marzo de 2010, desde http://www.rieoei.org/deloslectores/648Posada.PDF.

- Rodic, L.J. \& Sah, R.D. (2005). Professional Capacity Needs Assessment for Millennium Development Goal on Water Supply and Sanitation in Asia. XII World Water Congress of IWRA - Water for Sustainable Development Towards Innovative Solutions, 22-25 November 2005, New Delhi, India. - Restrepo, I. (2004). Tendencias mundiales en la gestión de recursos hídricos: desafíos para la ingeniería del agua. Ingeniería y competitividad, Vol. 6, No. 1. 
63-70 pp. Facultad de Ingeniería, Universidad del Valle, Cali. Rodríguez, M. (s.f.). La interdisciplinariedad: Acción comunicativa científica y humana. Centro de Servicios Pedagógicos. Obtenido en febrero de 2009, desde http://ayura.udea.edu.co/servicios/1_5.htm

- Sánchez, Myriam. (2007). Análisis del retorno a la inversión en la cooperación científico tecnológica: Una herramienta para la relación Ciencia-Sociedad en Colombia. Cooperación Internacional de Acción Social, Bogotá, 15 de marzo de 2007.

- Sánchez, V.A. (2002). Psicología social aplicada: teoría, método y práctica. Madrid. Editorial Pearson Educación. - Sotolongo, P.L., Delgado, C. J. (2006). La complejidad y el diálogo transdisciplinario de saberes. Capítulo IV. En publicación: La revolución contemporánea del saber y la complejidad social. Acceso al texto completo: http://bibliotecavirtual.clacso.org.ar/ar/libros/campus/soto/Capitulo\%20IV.p df

- Stokols, D. (2006). Toward a science of transdisciplinary action research. American Journal of Community Psychology, 38, 63-77. - Tiessen, H. (2008). Environmental Science and Society. The Bologna Centre Journal of International Affairs (http://bcjournal.org/2008). - Torres, J. (1994). Globalización e interdisciplinariedad: el currículum integrado. Jurjo Torres Santomé. Madrid Ediciones Morata. 72p. - Torres Santome, J. (1996). Globalización e interdisciplinariedad: El currículum integrado. Madrid: Morata, 1996. - Valle, Mónica. (2005). Comunicación: Disciplinariedad, Interdisciplinaridad y Transdiciplariedad. Revista Comunicologí@: indicios y conjeturas, Primera Época, Número 3, Primavera 2005, Publicación Electrónica del Departamento de Comunicación de la Universidad Iberoamericana Ciudad de México. Disponible en:

http://revistacomunicologia.org/index.php?option=com_content\&task=view \&id=95\&ltemid=89

- Van del Linde, G. (2007, Jul-Dic). ¿Por qué es importante la interdisciplinariedad en la educación superior? Cuadernos de Pedagogía Universitaria, Año 4. No. 8. 11-13. Pontificia Universidad Católica Madre y Maestra, Rep. Domin. - Vessuri, H. (2008). El futuro nos alcanza: Mutaciones previsibles de la Ciencia y la Tecnología. Capítulo 2. En Ana Lucía Gazzola y Axel Didriksson, (Eds.), Tendencias de la Educación Superior en América Latina y el Caribe. 410 pp. Caracas:

IESALC-UNESCO.

- Wallerstein, Immanuel et al. (1996). Open the social sciences (report of the Gulbenkian Comisión on the restructuring of social sciences). Stanford: Stanford University Press. - Zárate, CH. (2007, Jul-Dic). El reto de la interdisciplinariedad: desde su concepción hacia la práctica pedagógica. Cuadernos de Pedagogía Universitaria, Año 4. No. 8. 9-10. Pontificia Universidad Católica Madre y Maestra. Rep. Domin.

1. Ph.D. en Hidráulica y Medio Ambiente, Diploma de Estudios Avanzados en Hidráulica y Medio Ambiente, M.Sc en Suelos y Aguas - Universidad Nacional de Colombia. Ingeniero Agrícola - Universidad del Valle - Universidad Nacional de Colombia. Director Grupo de Investigación en Ingeniería de Recursos Hídricos y Suelos -IREHISA-, Profesor Escuela de Ingeniería de los Recursos Naturales y del Ambiente -EIDENAR-, Universidad del Valle, Colombia. 